\title{
Rapid Detection of K1 Hypervirulent Klebsiella pneumoniae by MALDI-TOF MS
}

\author{
Yonglu Huang ${ }^{1+}$, Jiaping $\mathrm{Li}^{1+}$, Danxia Gu${ }^{1}$, Ying Fang ${ }^{1}$, Edward W. Chan ${ }^{2,3}$, \\ Sheng Chen ${ }^{2,3 *}$ and Rong Zhang ${ }^{1 *}$
}

\begin{abstract}
${ }^{1}$ Department of Clinical Microbiology, Second Affiliated Hospital of Zhejiang University, Hangzhou, China, ${ }^{2}$ Shenzhen Key Lab for Food Biological Safety Control, Food Safety and Technology Research Center, Hong Kong PolyU Shenzhen Research Institute, Shenzhen, China, ${ }^{3}$ State Key Lab of Chirosciences, Department of Applied Biology and Chemical Technology, The Hong Kong Polytechnic University, Hong Kong, China
\end{abstract}

Hypervirulent strains of Klebsiella pneumoniae (hvKP) are genetic variants of K. pneumoniae which can cause life-threatening community-acquired infection in healthy individuals. Currently, methods for efficient differentiation between classic K. pneumoniae (CKP) and hvKP strains are not available, often causing delay in diagnosis and treatment of hvKP infections. To address this issue, we devised a Matrix-assisted laser desorption/ionization time-of-flight (MALDI-TOF) mass spectrometry (MS) approach for rapid identification of $\mathrm{K} 1$ hvKP strains. Four standard algorithms, genetic algorithm (GA), support vector machine (SVM), supervised neural network (SNN), and quick classifier $(\mathrm{QC})$, were tested for their power to differentiate between $\mathrm{K} 1$ and non-K1 strains, among which SVM was the most reliable algorithm. Analysis of the receiver operating characteristic curves of the interest peaks generated by the SVM model was found to confer highly accurate detection sensitivity and specificity, consistently producing distinguishable profiles for $\mathrm{K} 1 \mathrm{hvKP}$ and non-K1 strains. Of the $43 \mathrm{~K}$. pneumoniae modeling strains tested by this approach, all were correctly identified as K1 hvKP and non-K1 capsule type. Of the 20 non-K1 and $17 \mathrm{~K} 1$ hvKP validation isolates, the accuracy of K1 hvKP and non-K1 identification was 94.1 and $90.0 \%$, respectively, according to the SVM model. In summary, the MALDI-TOF MS approach can be applied alongside the conventional genotyping techniques to provide rapid and accurate diagnosis, and hence prompt treatment of infections caused by hvKP.

Keywords: K1 hvKP, MALDI-TOF MS, rapid detection, SVM model, typical spectra

\section{INTRODUCTION}

Klebsiella pneumoniae, a facultative anaerobic gram-negative bacillus (Fang et al., 2004), is an important opportunistic pathogen associated with both community-acquired and nosocomial infection such as pneumonia, urinary tract infections, septicemia, and wound infections, especially among patients in ICU (Vardakas et al., 2015). The first case of hypervirulent Klebsiella pneumoniae (HvKP) infection was reported to have originated from a patient with liver abscess in China in 1980s (Siu et al., 2012). Hvkp is a variant which is morphologically different from the classic strain in terms of appearance of colonies grown on agar plate. HvKP is not only able to cause nosocomial infection in immunocompromised patients, but more importantly, it often causes life-threatening 
community-acquired (CA) infection in healthy individuals, eliciting a great concern worldwide (Liu et al., 2014). In recent years, the incidence of hvKP infection has increased markedly in various countries including Asia (Zhang et al., 2014), Europe (Decré et al., 2011), and South America (Vila et al., 2011).

Different from classical K. pneumoniae (cKP), hvKP has high iron acquisition ability, an increase in capsule production mediated by $\mathrm{rmpA} / \mathrm{rmpA} 2$, which confers the hypermucoviscous, and association with Mucoviscosityassociated gene A (magA) and are commonly seen in K1, K2, K5, $\mathrm{K} 20$, K54, and $\mathrm{K} 57$ with $\mathrm{K} 1$ and $\mathrm{K} 2$ being the most dominant serotypes (Yeh et al., 2010; Shon et al., 2013). Emergence of hvKP strains represents a huge threat to human health (Shen et al., 2013) but methods for efficient differentiation between classic and hvKP strains are not available.

Matrix assisted laser desorption ionization-time of flight mass spectrometry (MALDI-TOF MS) is considered as an efficient tool which can accurately identify both commonly encountered pathogenic bacterial species and microbial pathogens that are difficult to identify, such as yeasts, anaerobes, and fastidious microorganisms (Martiny et al., 2012). Recently, some studies have shown that this technology exhibits the capacity for rapid discrimination of antibiotic resistant strains such as methicillin-resistant Staphylococcus aureus (Madhava Charyulu et al., 2012; Hu Yy et al., 2015) and carbapenemresistant Enterobacteriaceae (Lau et al., 2014) from the sensitive organisms, detection of virulence factors such as S. aureus delta-toxin (Gagnaire et al., 2012; Josten et al., 2014), and epidemiological typing (Josten et al., 2013). In this work, we developed a MALDI-TOF MS method for rapid identification of K1 K. pneumoniae isolates, and evaluated its reliability in rapid detection of major K. pneumoniae virulence factors.

\section{MATERIALS AND METHODS}

\section{Conventional K1 hvKP Identification}

A string test was performed to identify hvKP from clinical $K$. pneumoniae strains. A positive string test is defined as the formation of a mucoviscous string of $>5 \mathrm{~mm}$ in length when using a bacteriology inoculation loop to touch and stretch a colony grown overnight on an blood agar plate at $35^{\circ} \mathrm{C}$ (Fang et al., 2007). The capsular polysaccharide synthesis virulence genes (K1, K2, K5, K20, K54, and K57) and other relevant genes (wcaG, rmpA, magA, and Aerobactin) were amplified through a TPersonal cycler (Biometra, Germany) to further identify K1 hvKP strains. Primer sequence and annealing temperature were shown in Table 1. PCR products were sequenced using an ABI 3730 sequencer (Applied Biosystems, Foster City, CA); and the data obtained were compared with the reported sequences retrieved from GenBank. A strain with positive string test, positive K1 positive K1 capsular polysaccharide gene and one or more of the virulence was defined as hvKP.

\section{Molecular Typing}

Epidemiological relatedness of the $\mathrm{K} 1$ strains was studied by pulsed-field gel electrophoresis (PFGE) and multilocus sequence typing (MLST). Twenty-three K1-positive K. pneumoniae strains were genotyped by PFGE following the PulseNet protocol provided by the website of the U.S. Centers for Disease Control and Prevention (http://www.cdc.gov/pulsenet/pathogens/index. html). The bacterial cells were digested with the XbaI restriction

TABLE 1 | List of primers for detection of Klebsiella pneumoniae virulence genes.

\begin{tabular}{|c|c|c|c|c|c|}
\hline Gene & Primer & Primer sequence & Amplicon (bp) & Annealing temperature $\left({ }^{\circ} \mathrm{C}\right)$ & References \\
\hline \multirow[t]{2}{*}{ K1 } & K1-F & GTAGGTATTGCAAGCCATGC & 1047 & 55 & Fang et al., 2007 \\
\hline & K1-R & GCCCAGGTTAATGAATCCGT & & & \\
\hline \multirow[t]{2}{*}{ K2 } & K2-F & GACCCGATATTCATACTTGACAGAG & 641 & 57 & Turton et al., 2008 \\
\hline & $\mathrm{K} 2-\mathrm{R}$ & CCTGAAGTAAAATCGTAAATAGATGGC & & & \\
\hline K5 & K5-F & TGGTAGTGATGCTCGCGA & 280 & 55 & Turton et al., 2008 \\
\hline K20 & $\mathrm{K} 20-\mathrm{R}$ & GTTATACGATGCTCAGTCGC & & & \\
\hline \multirow[t]{2}{*}{ K54 } & K54-F & CATTAGCTCAGTGGTTGGCT & 881 & 55 & Fang et al., 2007 \\
\hline & $\mathrm{K} 54-\mathrm{R}$ & GCTTGACAAACACCATAGCAG & & & \\
\hline \multirow[t]{2}{*}{ K57 } & K57-F & CTCAGGGCTAGAAGTGTCAT & 1037 & 55 & Fang et al., 2007 \\
\hline & K57-R & CACTAACCCAGAAAGTCGAG & & & \\
\hline rmpA & $r m p A-R$ & CTTGCATGAGCCATCTTCA & & & \\
\hline \multirow[t]{2}{*}{$\operatorname{mag} A$} & magA-F & GGTGCTCTTTACATCATTGC & 1282 & 53 & Fang et al., 2004 \\
\hline & $\operatorname{mag} A-\mathrm{R}$ & GCAATGGCCATTTGCGTTAG & & & \\
\hline \multirow[t]{2}{*}{ Aerobactin } & Aerobactin-F & GCATAGGCGGATACGAACAT & 556 & 55 & Yu et al., 2008 \\
\hline & Aerobactin-R & CACAGGGCAATTGCTTACCT & & & \\
\hline
\end{tabular}


enzyme and were separated in a Rotaphor System 6.0 instrument (Whatman Biometra). Banding patterns of 23 isolates were analyzed using the UVIBand software program (UVItec Ltd., Cambridge, United Kingdom), and the degree of sequence homology was calculated (unweighted pair group method using average linkages [UPGMA],0.5\% Master Lane). MLST was performed on representative isolates of each clonal type. Seven housekeeping genes of $K$. pneumoniae (gapA, infB, $m d h$, pgi, phoE, $r p o B$, and ton $B$ ) were amplified, sequenced and analyzed (Turton et al., 2007). Allele sequences and sequence types (STs) were analyzed according to the MLST database (http://pubmlst. $\operatorname{org} /)$.

\section{Protein Extraction}

K.pneumoniae isolates were inoculated onto Columbia blood agar (Oxoid, Cambridge, UK) containing 5\% sheep blood and incubated $18-24 \mathrm{~h}$ at $35^{\circ} \mathrm{C}$. Several uniform colonies from fresh plates were re-suspended into $300 \mu \mathrm{l}$ of distilled water. After addition of $900 \mu$ l ethanol, the extraction tube was centrifuged at $12,000 \times \mathrm{g}$ for $2 \mathrm{~min}$, the supernatant was then discarded. The bacterial pellet was re-suspended in $50 \mu \mathrm{l} 70 \%$ formic acid. Before centrifuging again, $50 \mu \mathrm{l}$ acetonitrile was added. After 2 min centrifugation at $12,000 \times$ g, $1 \mu \mathrm{l}$ of supernatant was spotted onto the ground steel target and dried at room temperature. One microliter of alphacyano-4-hydroxycinnamic acid (CHCA) was overlaid and dried again.

\section{MALDI-TOF MS Analysis}

MALDI-TOF MS analysis was performed on a Bruker MicroFlex LT mass spectrometer (Bruker Daltonics). Spectra were acquired according to the manufacturer's recommendations, mass range was from 2000 to $20,000 \mathrm{Da}$ and the laser intensity was kept constant. Mass spectra were analyzed by the Biotyper 3.0 software and library (version 3.0, Bruker Daltonics). Identification score criteria used followed those recommended by the manufacturer: a score of $>2.000$ indicated species-level identification, a score of 1.700-1.999 indicated identification to the genus level, and a score of $<1.700$ was interpreted as inconclusive.

\section{Data Analysis}

The ClinProTools software (v3.0; Bruker Daltonics) was used for peak analysis. Models were generated using all four available algorithms, genetic algorithm (GA), support vector machine (SVM), supervised neural network (SNN), and quick classifier (QC), followed by comparison to each other. For each model, the recognition capability and cross validation were calculated to demonstrate the sensitivity and specificity of the model, statistical analysis were obtained by the most reliable algorithm model. The receiver operating characteristic (ROC) curves for each of the peaks of interest were obtained from the ClinProTools software. The area under curve (AUC) was used to evaluate the performance of each algorithm.

\section{RESULTS}

\section{Bacterial Isolates Used in This Study}

Forty K1hvKP strains were identified from 438 clinical nonrepeated $K$. pneumoniae strains isolated from patients in Zhejiang Provincial hospital. Twenty-three out of the $40 \mathrm{~K} 1$ hvKP and 20 non-K1 isolates were randomly chosen for the development of MALDI-TOF MS method. The rest of $17 \mathrm{~K} 1$ isolates along with another 20 non-K1 isolates were used to evaluate the differentiation power of the MALDI-TOF MS method.

\section{Molecular Features of K1 and Non-K1 Strains}

All $40 \mathrm{~K} 1$ hvKP contained K1 capsular gene and at least magA and Aerobactin genes. MLST analysis identified four STs including ST23, ST520, ST700, and ST1552 with ST23 the most dominant one (20 ST23 out of a total of $23 \mathrm{~K} 1 \mathrm{hvKP}$ ). In addition to magA and Aerobactin genes, all ST23 K1 hvKP also contained wcaG and $r m p A$; one ST700 contained $w c a G$ (Table 2). Within the 20 ST23 K1 hvKP strains, only two (21 and 23) were shown to be identical by PFGE analysis (Figure 1). In contrast, the non-K1 strains are found to exhibit a wide range of genetic diversity with 14 different STs being identified in the 20 non-K1 K. pneumoniae. None of the non-K1 K. pneumoniae harbored any of the four genetic markers suggesting they are not hvKP strains (Table 2). Notably, STs in K1 hvKP and non-K1 K. pneumoniae were different suggesting the close association of STs to K1 hvKP in particular ST23.

TABLE 2 | Prevalence of ST types and known virulence genes in K1 hvKP and non-K1 K. pneumoniae strains.

\begin{tabular}{|c|c|c|c|c|c|c|}
\hline \multirow{2}{*}{$\begin{array}{l}\text { Capsule } \\
\text { type }\end{array}$} & \multirow{2}{*}{$\begin{array}{c}\mathrm{ST} \\
\text { types }\end{array}$} & \multirow{2}{*}{$\begin{array}{c}\text { No. of } \\
\text { isolates }\end{array}$} & \multicolumn{4}{|c|}{ Other virulence genes } \\
\hline & & & wcaG & $r m p A$ & $\operatorname{mag} A$ & Aerobactin \\
\hline \multirow[t]{4}{*}{ K1 } & 23 & 20 & + & + & + & + \\
\hline & 520 & 1 & - & - & + & + \\
\hline & 700 & 1 & + & - & + & + \\
\hline & 1552 & 1 & - & - & + & + \\
\hline \multirow[t]{14}{*}{ non-K1 } & 12 & 1 & - & - & - & - \\
\hline & 34 & 1 & - & - & - & - \\
\hline & 35 & 4 & - & - & - & - \\
\hline & 36 & 1 & - & - & - & - \\
\hline & 37 & 2 & - & - & - & - \\
\hline & 138 & 2 & - & - & - & - \\
\hline & 705 & 1 & - & - & - & - \\
\hline & 753 & 1 & - & - & - & - \\
\hline & 875 & 1 & - & - & - & - \\
\hline & 983 & 1 & - & - & - & - \\
\hline & 1411 & 1 & - & - & - & - \\
\hline & 1547 & 2 & - & - & - & - \\
\hline & 1548 & 1 & - & - & - & - \\
\hline & 1551 & 1 & - & - & - & - \\
\hline
\end{tabular}




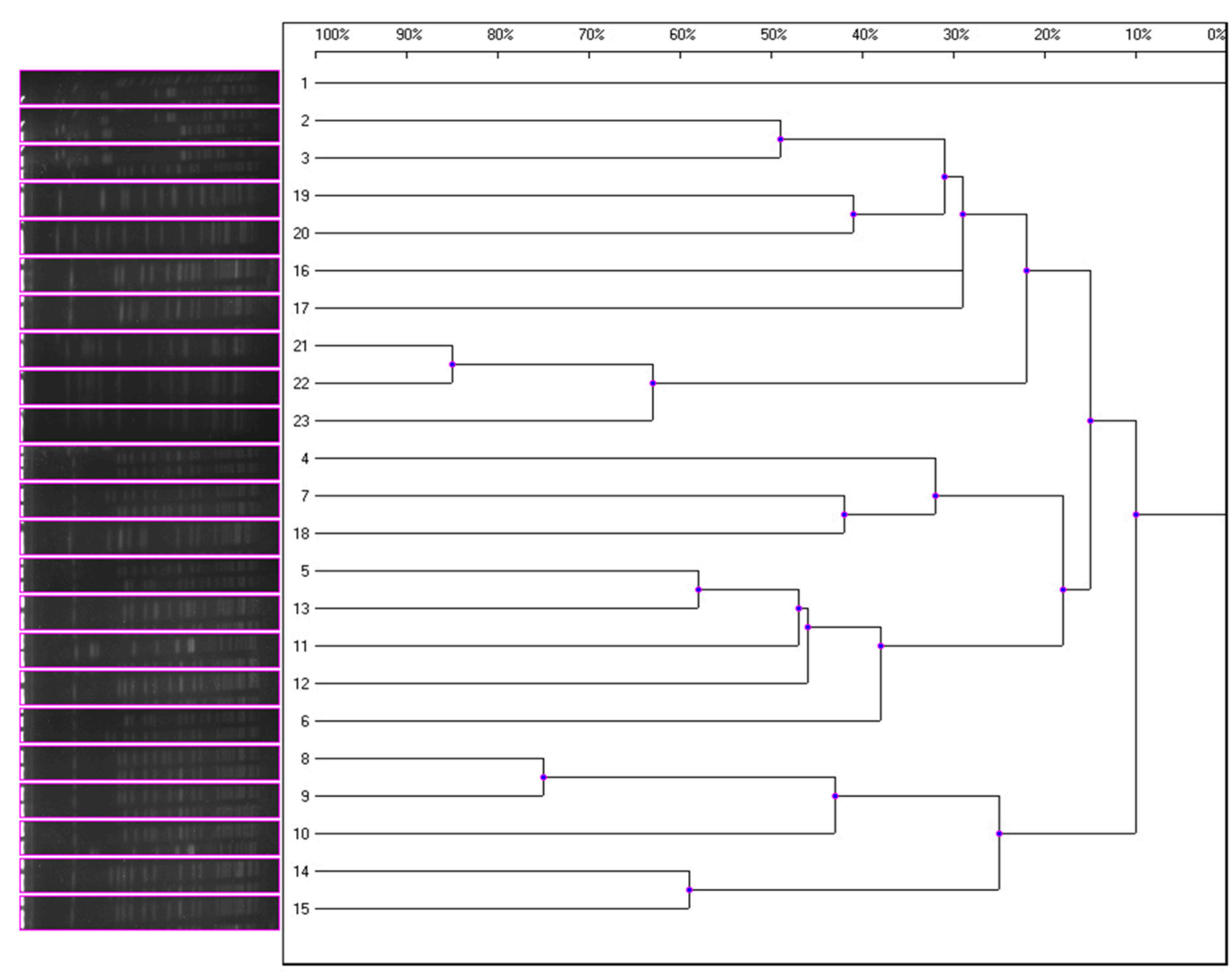

FIGURE 1 | Magnified dendrogram (representation of hierarchical cluster analysis) of $23 \mathrm{~K} 1 \mathrm{hvKP}$ isolates.

\section{Data Analysis by MALDI-TOF MS}

Of the $43 \mathrm{~K}$. pneumoniae modeling isolates analyzed, all (100\%) were identified correctly as K. pneumoniae by MALDI-TOF MS. The models generated using four standard algorithms, GA, SVM, $\mathrm{SNN}$, and QC, for comparing K1 with non-K1 isolates, were shown in Table 3. The use of the QC standard algorithm resulted in the lowest scores, whereas GA standard algorithm exhibited the highest specificity (recognition capability at 100\%) when compared to the SVM standard algorithm (recognition capability at $97.83 \%$ ). Yet SVM gave the highest sensitivity at $83.45 \%$ among four models, which is higher than the rate of $73.87 \%$ for GA. However, the specificity of SVM is slightly lower than GA being at $97.83 \%$. Overall, SVM and SNN were the most reliable model for differentiating between $\mathrm{K} 1$ from classic or non-K1 isolates. The study showed that peaks or integration regions chosen for differentiation of K1 K. pneumoniae status by all four of the models were similar. The important peaks identified by the SVM model for K1 were 14, 31, 33, and 34. The peak statistics for these peaks are shown in Table 4.

The low $P$-values for the Anderson-Darling test (PAD) are evidence of the abnormal distribution of the data obtained. Therefore, the $P$-value of the PWKW ( $P$-value from combined Wilcoxon rank-sum test and Kruskal-Wallis test) is preferred over the PTTA ( $p$-value of $t$-test) (as this is preferable for normally distributed data). The low $P$-values obtained from
PWKW (all were <0.05) indicated that the observed intensity differences of the individual peaks are highly statistically significant (i.e., the lower the $P$-value is, the higher the chance that a respective peak signal is suited to differentiate between the two classes) (Table 4).

The receiver operating characteristic (ROC) curves for each of the peaks of interest generated by the SVM model were also obtained from the ClinProTools software. The area under curve (AUC) can reflect the confidence level of each peak in identifying the sensitivity and specificity of virulent strains and non-K1 strains group, with an AUC of 0.5 representing purely random chance and an AUC of 1 indicating a perfect test (100\% sensitivity and specificity; Table 4$)$. All characteristic peaks used for distinguishing virulent from non-K1 strains were $>0.8$, confirming that these peaks can be used to differentiate $\mathrm{K} 1$ from non-K1 $K$. pneumoniae isolates with high accuracy. To demonstrate the differences visually, four representative peaks are shown in Figure 2, in which the spectra of the $\mathrm{K} 1$ and non-K1 $K$. pneumoniae isolates were distinguishable.

\section{Prospective Validation}

Another 20 randomly selected non-K1 K. pneumoniae and 17 remaining K1-positive hvKP isolates were chosen to perform the prospective verification. The verification result indicated that the 
TABLE 3 | Specificity and sensitivity of different algorithms models for differentiation between K1 and non-K1 K. pneumoniae isolates.

\begin{tabular}{|c|c|c|c|c|c|c|}
\hline \multirow[t]{2}{*}{ Algorithms model } & \multicolumn{2}{|c|}{ Assay development } & \multicolumn{4}{|c|}{ Assay validation } \\
\hline & $\begin{array}{c}\text { Cross } \\
\text { validation }\end{array}$ & $\begin{array}{c}\text { Recognition } \\
\text { capability }\end{array}$ & $\begin{array}{c}\text { Specificity } \\
\text { (true negative) }\end{array}$ & $\begin{array}{l}95 \% \mathrm{Cl} \text { of } \\
\text { Specificity }\end{array}$ & $\begin{array}{c}\text { Sensitivity } \\
\text { (true positive) }\end{array}$ & $\begin{array}{l}95 \% \mathrm{Cl} \text { of } \\
\text { Sensitivity }\end{array}$ \\
\hline GA & $100 \%$ & $73.9 \%$ & 16/17 (94.1\%) & $86.5-100 \%$ & 17/20 (85.0\%) & 73.5-96.5\% \\
\hline SVM & $97.8 \%$ & $83.5 \%$ & $16 / 17(94.1 \%)$ & $86.5-100 \%$ & 18/20 (90.0\%) & 80.3-99.7\% \\
\hline SNN & $100 \%$ & $81.4 \%$ & 16/17 (94.1\%) & $86.5-100 \%$ & 18/20 (90.0\%) & 80.3-99.7\% \\
\hline QC & $85.7 \%$ & $70.7 \%$ & 15/17 (88.3\%) & $77.9-98.6 \%$ & 16/20 (80.0\%) & $67.1-92.9 \%$ \\
\hline
\end{tabular}

TABLE 4 | ClinProTools peak statistics for the four peaks of interest in both K1 hvKP and non-K1 K. pneumoniae*.

\begin{tabular}{|c|c|c|c|c|c|c|c|c|c|c|c|}
\hline Peak & Mass & DAve & PTTA & PWKW & PAD & Ave1 & Ave2 & SD1 & SD2 & CV1 & CV2 \\
\hline 14 & 3586.58 & 2.1 & 0.000339 & 0.000258 & 0.0729 & 3.1 & 5.2 & 1.23 & 1.48 & 39.71 & 28.36 \\
\hline 31 & 4744.66 & 8.27 & 0.000164 & 0.000258 & 0.0843 & 12.91 & 21.18 & 5.41 & 4.61 & 41.94 & 21.74 \\
\hline 33 & 5044.84 & 1.62 & 0.00036 & 0.000819 & 0.225 & 3.07 & 4.69 & 1.34 & 0.83 & 43.6 & 17.77 \\
\hline 34 & 5148.93 & 2.59 & 0.000198 & 0.000525 & 0.0729 & 3.53 & 6.12 & 1.51 & 1.68 & 42.72 & 27.49 \\
\hline
\end{tabular}

*Sort mode, delta average arithmetic; peak, peak index; mass, $\mathrm{m} / \mathrm{z}$ value; $D A v e$, difference between the maximal and the minimal average peak area/intensity of all classes; $P$ TTA, $P$-value of t-test; PWKW, P-value of Wilcoxon (preferable for abnormally distributed data); PAD, P-value of Anderson-Darling test (range, 0-1; 0, abnormally distributed; 1, normally distributed);

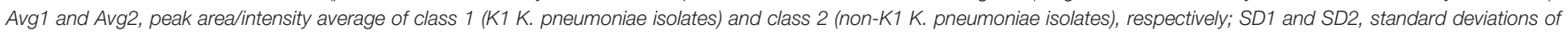
the peak area/intensity average of class 1 and class 2, respectively; CV1 and CV2, coefficient of variation (in percentage) of class 1 and class 2, respectively.
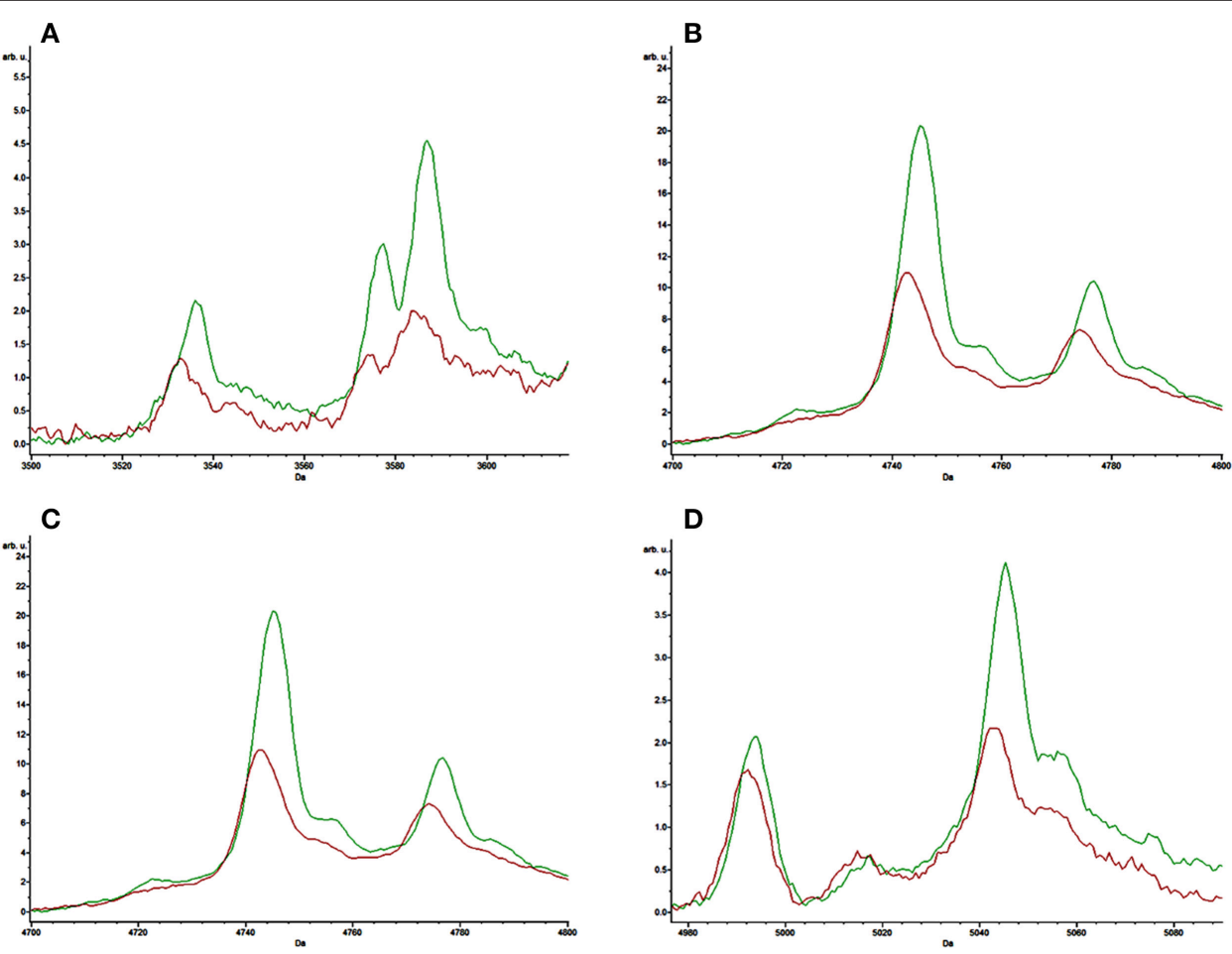

FIGURE 2 | Representative comparison of the average spectra of the K1 hvKP isolates (red) and non-K1 K. pneumoniae isolates (green). (A-D) are the representative spectra of $\mathrm{K} 1 \mathrm{hvKP}$ and non-K1 K. pneumoniae.

accuracy of K1 and non-K1 identification was 94.1 and 90.0\%, respectively, according to the SVM model suggesting the high accuracy of MALDI-TOF MS method to rapid identification of K1 hvKP (Table 3).

\section{DISCUSSION}

Hospital-acquired $K$. pneumoniae clinical isolates normally exhibit relatively low-level virulence, whereas in most cases of 
community-acquired pneumonia, Klebsiella isolates are generally highly virulent and known to produce mucoid colonies. Recent studies indicated that hvKP induced liver abscess was a new type of invasive infections (Siu et al., 2012), and that hvKP can not only cause liver abscess, but also metastatic infections such as bacteremia, meningitis, endophthalmitis, and necrosis fasciitis, often resulting in fatality in severe cases. A number of virulence factors have been identified in pathogenic K. pneumoniae, including capsular polysaccharide, mucus related gene A (mucoviscosity-associated gene A, magA), mucous phenotype A regulator gene (regulator of mucoid phenotype gene A, rmpA), and, aerobactin. Capsular polysaccharide is considered one of the major contributive factors of virulence in K. pneumoniae, promoting biofilm formation and exhibiting anti-opsonin effect which can help combat the host immune response when expressed in the host body (Cortés et al., 2002). To date, K. pneumoniae producing K1 type capsule is the major pathogen causing community-acquired lung infections in Asia (Lin et al., 2015).

Current hvKP detection methods include PCR amplification, multilocus sequence typing (MLST), pulsed field gel electrophoresis (PFGE), and the proteomics approach, which are complicated and time consuming, and demand technical competency. Since hvKP is highly virulent, causing high mobility and mortality among infected patients, a rapid and accurate clinical identification method is urgently needed to guide proper treatment of patients infected by hvKP. MALDI-TOF MS is a revolutionary technique for clinical bacteria identification. It has high power to identify clinical common bacteria, yeasts and fungi and has been shown to be used to identify antimicrobial resistance and virulence gene products. Based on the specific peaks, MALDI TOF mass spectrometry can be used to identify bla $a_{\mathrm{KPC}}$-positive $K$. pneumoniae, van B positive Enterococci feces and virulence factors of $S$. aureus such as delta-toxin and PSM-mec (Josten et al., 2014).

\section{REFERENCES}

Cortés, G., Borrell, N., de Astorza, B., Gómez, C., Sauleda, J., and Alberti, S. (2002). Molecular analysis of the contribution of the capsular polysaccharide and the lipopolysaccharide $\mathrm{O}$ side chain to the virulence of Klebsiella pneumoniae in a murine model of pneumonia. Infect. Immun. 70, 2583-2590. doi: 10.1128/IAI.70.5.2583-2590.2002

Decré, D., Verdet, C., Emirian, A., Le Gourrierec, T., Petit, J. C., Offenstadt, G., et al. (2011). Emerging severe and fatal infections due to Klebsiella pneumoniae in two university hospitals in France. J. Clin. Microbiol. 49, 3012-3014. doi: 10.1128/JCM.00676-11

Fang, C. T., Chuang, Y. P., Shun, C. T., Chang, S. C., and Wang, J. T. (2004). A novel virulence gene in Klebsiella pneumoniae strains causing primary liver abscess and septic metastatic complications. J. Exp. Med. 199, 697-705. doi: 10.1084/jem.20030857

Fang, C. T., Lai, S. Y., Yi, W. C., Hsueh, P. R., Liu, K. L., and Chang, S. C. (2007). Klebsiella pneumoniae genotype K1: an emerging pathogen that causes septic ocular or central nervous system complications from pyogenic liver abscess. Clin. Infect. Dis. 45, 284-293. doi: 10.1086/519262

Gagnaire, J., Dauwalder, O., Boisset, S., Khau, D., Freydière, A. M., Ader, F., et al. (2012). Detection of Staphylococcus aureus delta-toxin production by whole-cell MALDI-TOF mass spectrometry. PLoS ONE 7:e40660. doi: 10.1371/journal.pone. 0040660
In this study, we used 20 non-K1 K. pneumoniae isolates with various ST patterns as control strains. Despite exhibiting different MS spectra, however, we were still able to differentiate between the non-K1 K. pneumoniae and $\mathrm{K} 1$ hvKP strains, producing results which were highly consistent with data of MLST tests and genotyping of known virulence genes. Although no specific spectrum has been identified for K1 hvKP, four peaks at $\mathrm{m} / z$ of $3587,4745,5045$, and 5149 were shown to be significantly $(P<0.05)$ stronger among the non-K1 strains when compared to the K1 hvKP strains. In addition, ROC of AUC reached $>0.8$, suggesting that these parameters have high predictive value for distinguishing between $\mathrm{K} 1$ and non-K1 K. pneumoniae. Since these peaks could also be reproduced in other clinical validation isolates with similar accuracy and sensitivity, we conclude that MALDI-TOF MS based method is a simple approach for rapidly and accurately identifying K1 K. pneumoniae, which have become prevalent and clinically significant, thereby greatly facilitating prompt and efficient treatment of infections caused by this notorious pathogen. The limitation of current study includes the 94.1 and $90.0 \%$ discrimination power for $\mathrm{K} 1 \mathrm{hvKP}$ and non-K1 $\mathrm{KP}$, respectively, which may result in some false positive results, and relatively small number of isolates for assay development and validation, increasing of which may significantly improve the accuracy and specificity of the method. The study at this stage is only a preliminary investigation demonstrating the possibility to detect K1 hvKP by MALDI-TOF MS and requires extensive clinical validation before it can be used as a validated clinical method.

\section{ACKNOWLEDGMENTS}

This work was supported by the Chinese National Key Basic Research and Development Program (2013CB127200) and Science and Technology Department of Zhejiang Province (2014C33191).

Hu Yy, C. J., Zhou, H. W., Jiang, Y., and Zhang, R. (2015). Rapid identification of methicillin-resistant Staphylococcus aureus and methicillin-sensitive Staphylococcus aureus strains by matrix-assisted laser desorption/ionization timeof-flight mass spectrometry. Chin. J. Microbiol. Immunol. 1, 42-45. doi: 10.3760/cma.j.issn.0254-5101.2015.01.009

Josten, M., Dischinger, J., Szekat, C., Reif, M., Al-Sabti, N., Sahl, H. G., et al. (2014). Identification of agr-positive methicillin-resistant Staphylococcus aureus harbouring the class A mec complex by MALDI-TOF mass spectrometry. Int. J. Med. Microbiol. 304, 1018-1023. doi: 10.1016/j.ijmm.2014.07.005

Josten, M., Reif, M., Szekat, C., Al-Sabti, N., Roemer, T., Sparbier, K., et al. (2013). Analysis of the matrix-assisted laser desorption ionization-time of flight mass spectrum of Staphylococcus aureus identifies mutations that allow differentiation of the main clonal lineages. J. Clin. Microbiol. 51, 1809-1817. doi: 10.1128/JCM.00518-13

Lau, A. F., Wang, H., Weingarten, R. A., Drake, S. K., Suffredini, A. F., Garfield, M. K., et al. (2014). A rapid matrix-assisted laser desorption ionization-time of flight mass spectrometry-based method for single-plasmid tracking in an outbreak of carbapenem-resistant Enterobacteriaceae. J. Clin. Microbiol. 52, 2804-2812. doi: 10.1128/JCM.00694-14

Lin, Y. T., Wang, Y. P., Wang, F. D., and Fung, C. P. (2015). Community-onset Klebsiella pneumoniae pneumonia in Taiwan: clinical features of the disease and associated microbiological characteristics of isolates from pneumonia and nasopharynx. Front. Microbiol. 9:122. doi: 10.3389/fmicb.2015.00122 
Liu, Y. M., Li, B. B., Zhang, Y. Y., Zhang, W., Shen, H., Li, H., et al. (2014). Clinical and molecular characteristics of emerging hypervirulent Klebsiella pneumoniae bloodstream infections in mainland China. Antimicrob. Agents Chemother. 58, 5379-5385. doi: 10.1128/AAC.02523-14

Madhava Charyulu, E., Gnanamani, A., and Mandal, A. B. (2012). Identification and discrimination of Methicillin Resistant Staphylococcus aureus strains isolated from burn wound sites using PCR and authentication with MALDITOF-MS. Indian J. Microbiol. 52, 337-345. doi: 10.1007/s12088-011-0245-8

Martiny, D., Busson, L., Wybo, I., El Haj, R. A., Dediste, A., and Vandenberg, O. (2012). Comparison of the Microflex LT and Vitek MS systems for routine identification of bacteria by matrix-assisted laser desorption ionizationtime of flight mass spectrometry. J. Clin. Microbiol. 50, 1313-1325. doi: 10.1128/JCM.05971-11

Nadasy, K. A., Domiati-Saad, R., and Tribble, M. A. (2007). Invasive Klebsiella pneumoniae syndrome in North America. Clin. Infect. Dis. 45, e25-e28. doi: $10.1086 / 519424$

Shen, D. X., Wang, J., and Li, D. D. (2013). Klebsiella pneumoniae liver abscesses. Lancet Infect. Dis. 13, 390-391. doi: 10.1016/S1473-3099(13)70068-9

Shon, A. S., Bajwa, R. P., and Russo, T. A. (2013). Hypervirulent (hypermucoviscous) Klebsiella pneumoniae: a new and dangerous breed. Virulence 4, 107-118. doi: 10.4161/viru.22718

Siu, L. K., Yeh, K. M., Lin, J. C., Fung, C. P., and Chang, F. Y. (2012). Klebsiella pneumoniae liver abscess: a new invasive syndrome. Lancet Infect. Dis. 12, 881-887. doi: 10.1016/S1473-3099(12)70205-0

Turton, J. F., Baklan, H., Siu, L. K., Kaufmann, M. E., and Pitt, T. L. (2008). Evaluation of a multiplex PCR for detection of serotypes K1, K2 and K5 in Klebsiella sp. and comparison of isolates within these serotypes. FEMS Microbiol. Lett. 284, 247-252. doi: 10.1111/j.1574-6968.2008.01208.x

Turton, J. F., Englender, H., Gabriel, S. N., Turton, S. E., Kaufmann, M. E., and Pitt, T. L. (2007). Genetically similar isolates of Klebsiella pneumoniae serotype K1 causing liver abscesses in three continents. J. Med. Microbiol. 56, 593-597. doi: 10.1099/jmm.0.46964-0

Turton, J. F., Perry, C., Elgohari, S., and Hampton, C. V. (2010). PCR characterization and typing of Klebsiella pneumoniae using capsular type-specific, variable number tandem repeat and virulence gene targets J. Med. Microbiol. 59, 541-547. doi: 10.1099/jmm.0.015198-0

Vardakas, K. Z., Matthaiou, D. K., Falagas, M. E., Antypa, E., Koteli, A., and Antoniadou, E. (2015). Characteristics, risk factors and outcomes of carbapenem-resistant Klebsiella pneumoniae infections in the intensive care unit. J. Infect. 70, 592-599. doi: 10.1016/j.jinf.2014.11.003

Vila, A., Cassata, A., Pagella, H., Amadio, C., Yeh, K. M., Chang, F. Y., et al. (2011) Appearance of Klebsiella pneumoniae liver abscess syndrome in Argentina: case report and review of molecular mechanisms of pathogenesis. Open Microbiol. J. 5, 107-113. doi: 10.2174/1874285801105010107

Yeh, K. M., Lin, J. C., Yin, F. Y., Fung, C. P., Hung, H. C., Siu, L. K., et al. (2010). Revisiting the importance of virulence determinant magA and its surrounding genes in Klebsiella pneumoniae causing pyogenic liver abscesses: exact role in serotype K1 capsule formation. J. Infect. Dis. 201, 1259-1267. doi: $10.1086 / 606010$

Yu, W. L., Ko, W. C., Cheng, K. C., Lee, C. C., Lai, C. C., and Chuang, Y. C. (2008) Comparison of prevalence of virulence factors for Klebsiella pneumoniae liver abscesses between isolates with capsular $\mathrm{K} 1 / \mathrm{K} 2$ and non-K1/K2 serotypes. Diagn. Microbiol. Infect. Dis. 62, 1-6. doi: 10.1016/j.diagmicrobio.2008.04.007

Zhang, Y., Ma, Y., Ye, L., Luo, Y., and Yang, J. (2014). Prevalence and antimicrobial susceptibility of hypervirulent Klebsiella pneumoniae isolates in China. Clin. Infect. Dis. 58, 1493-1494. doi: 10.1093/cid/ciu110

Conflict of Interest Statement: The authors declare that the research was conducted in the absence of any commercial or financial relationships that could be construed as a potential conflict of interest.

Copyright (c) 2015 Huang, Li, Gu, Fang, Chan, Chen and Zhang. This is an openaccess article distributed under the terms of the Creative Commons Attribution License (CC BY). The use, distribution or reproduction in other forums is permitted, provided the original author(s) or licensor are credited and that the original publication in this journal is cited, in accordance with accepted academic practice. No use, distribution or reproduction is permitted which does not comply with these terms. 